\title{
EL ENTRENAMIENTO MULTILATERAL ES LA BASE DE HABILIDADES TEMPRANAS TRANSFERIBLES Y SIN RIESGO EN NIÑOS.
}

\author{
MULTILATERAL TRAINING IS THE BASIS OF EARLY TRANSFERABLE SKILLS AND \\ WITHOUT RISK IN CHILDREN.
}

\section{Mauricio Sanabria}

Especialista en Entrenamiento Deportivo, UDCA. (2)

Doctor en Ciencias de la Actividad Física y del

Deporte, profesor de la UDCA.

E-mail:

\section{Daniel Oliveros}

Doctor en Ciencias de la Actividad Física y el Deporte de León, España.

E-mail: danieloliverosw@hotmail.com

\section{RESUMEN}

Este estudio proporciona una visión general de la práctica de la diversificación deportiva temprana o entrenamiento multilateral y los beneficios potenciales asociados a esto. El objetivo principal de esta revisión consiste en investigar la literatura sobre el efecto del entrenamiento multilateral en el desarrollo de habilidades bio-motoras y los mecanismos para incidir en la disminución de riesgos psíquicos, físicos y sociales asociados a la especialización deportiva temprana. Se efectuó una revisión sistemática de artículos relacionados a la diversificación y especialización deportiva temprana. Se identificaron 66 artículos a través de la búsqueda en las bases de datos Sportdiscuss y Google Scholar; se llevó a cabo entre los meses de septiembre de 2017 y agosto de 2018. Para la selección de los artículos se consideró la relevancia y utilidad del tema y la credibilidad o experiencia de los autores en el campo. Los resultados de la revisión ponen en manifiesto la importancia del entrenamiento multilateral como base de habilidades tempranas transferibles y como la especialización temprana presenta una serie de riesgos y no es garantía para llegar al deporte de elite.

Palabras clave: Especialización temprana, entrenamiento multilateral, habilidades biomotoras, diversificación temprana, niños deportistas.

\section{ABSTRACT}

This study provides an overview of the practice of early sport sampling or multilateral training and the potential benefits associated with it. The main objective of this review is to investigate the literature on the effect of multi-training in the development of bio-motor skills and the mechanisms to influence the reduction of psychic, physical and social risks associated with early sports specialization. A systematic review of articles related to diversification and early sports specialization were carried out. 67 articles were identified through the search in the databases Sportdiscus and Google Scholar; It was carried out between the months of September 2017 and August 2018. 
For the selection of articles, the relevance and usefulness of the topic and the credibility or experience of the authors in the field were considered. The results of the review highlight the importance of multilateral training as a basis for early transferable skills and how early specialization presents multiple risks and is not a guarantee to reach elite in sport.

Key words: early sports specialization, unilateral training, multisport athletes, biomotor skills, Young athletes

\section{INTRODUCCIÓN}

El deporte de altos logros (alto rendimiento) requiere cada vez más deportistas con mayores cualidades genéticas, a los que se les pueda aplicar en mayor medida cargas altas de entrenamiento (volumen e intensidad), y de la misma forma, un óptimo desarrollo de sus capacidades físicas (velocidad, fuerza, resistencia y flexibilidad) y un amplio repertorio de habilidades motrices.

Por tal motivo, la especialización deportiva ha crecido durante los últimos 15 años, con la creencia que conducirá a oportunidades en deportes profesionales (Feeley \& cols., 2015), citados por Sluder, B. (2017). La creencia que se ha extendido entre padres, entrenadores y los propios niños, es que entre más jóvenes los niños participen en un solo deporte, mayores probabilidades de éxito tendrán en el mismo.

El desarrollo del talento y la experticia deportiva, no es sólo el resultado de práctica deliberada por un periodo superior a 10 años (Ericsson \& cols., 1993), citados por Jones y Petlichkoff (2008). La práctica deliberada tiene como propósito mejorar el rendimiento y es típicamente, monitoreada por un entrenador. Las exigencias de participar en este tipo de práctica por deportistas élite con altos niveles de rendimientos deberían ser aprobados con precaución (Jones y Petlichkoff, 2008).

Por tales motivos, el entrenamiento en edades tempranas debería acercarse al modelo de juego deliberado, que consiste en una práctica más divertida, multideportiva y con menos reglas rígidas.

\section{Justificación}

El proceso de dosificación del entrenamiento en niños y adolescentes, no se debe tomar a la ligera, porque los niños no son adultos en miniatura, sino seres en constante evolución. Por tal motivo, la planificación del entrenamiento en estas etapas, debería reconocer los estadios de desarrollo y crecimiento de los individuos. Cada etapa responde una serie de características biológicas que no deben pasarse por alto, esto se traduce en que las actividades físicas planteadas se deben acoplar a los niños y no someterlos a entrenamientos que sobrepasen sus capacidades.

Para que este proceso se lleve a cabo de la mejor medida hay que respetar el desarrollo biológico y motriz de los niños, teniendo en cuenta el punto de vista médico y pedagógico respectivamente. Hay que considerar que los niños son seres en proceso de crecimiento y que las tareas motrices se deben adaptar a su periodo evolutivo y no en el sentido contrario. Por tal motivo es de suma relevancia evaluar el efecto de la especialización deportiva precoz en relación con el sistema musculoesquelético, la personalidad de los jóvenes y 
el control de la incidencia de lesiones a temprana edad.

De igual manera Weiss (2015), en su artículo "Bo Knows", citando a Goodway y Robinson, concluye que, los niños en escuela primaria necesitan desarrollar una amplia base de habilidades motrices, experimentando diversas actividades, antes de elegir, si desean, un solo deporte en la adolescencia. Esta trayectoria de desarrollo podría maximizar la maestría de habilidades que satisfagan necesidades e intereses únicos de todos los niños y podrían facilitar la participación en muchas actividades físicas a lo largo de la vida. Adicionalmente, aunque puede ser necesaria la especialización temprana para alcanzar la excelencia y el pico de rendimiento en el deporte, hay evidencia que sugiere que su práctica deliberada no garantiza éxito deportivo. Además, hay estudios que muestran que niños que se especializan temprano, tienen más riesgo de lesiones por sobreuso, pueden no alcanzar todo su potencial de crecimiento y tampoco podrían transferir sus habilidades de manera positiva en la edad adulta.

\section{Problema}

Algunos estudios encuentran una estrecha relación entre la especialización temprana en el deporte y el aumento de lesiones en niños menores de 15 años. Y no solo esto, sino que podría ser sinónimo de agotamiento, perdida del disfrute y de la motivación.

Dentro de los mecanismos que refieren la aparición de lesiones, se encuentran el sobreuso, la inadecuada técnica y tiempos muy cortos o ausencias de descanso, en algunos casos de entrenamiento deportivo prematuro en niños y jóvenes.
De manera inversa niños que practican varios deportes de manera simultánea, reflejan una disminución en episodios lesivos.

No hay que desconocer, que hay deportes que requieren una iniciación temprana, como es el caso de la natación y la gimnasia, pero las etapas iniciales de inmersión en estos deportes debe responder a un trabajo multilateral y multideportivo, que permita ampliar el repertorio de cualidades biomotoras, formarse personalmente, sentar las bases de logros deportivos futuros $y$ disminuir la probabilidad de ausentarse de la práctica deportiva, ya sea por lesiones por sobreuso, agotamiento o por pérdida del disfrute y de la motivación.

Tal parece que la especialización temprana, entendida como la participación regular (sobre una base anual), en la que se concentra el desarrollo, entrenamiento y competencias de ese deporte específico, en edades inferiores a los 13 años, tiene consecuencias negativas asociadas. Se cree que al practicar un deporte en el que se efectúan una cantidad limitada de movimientos inherentes a su práctica, tiene el potencial de limitar el desarrollo de las habilidades motrices, lo que a su vez, podría afectar a largo plazo la probabilidad de participar en actividades físicas alternativas.

De modo contrario, el entrenamiento multilateral o participar en varios deportes (sampling) durante la niñez incrementa la probabilidad de desarrollar habilidades "prerrequisito", que pueden contribuir al desarrollo de muchas habilidades. Este es un mecanismo, por el cual, pueda ocurrir la transferencia de conocimiento, y es consistente, con la idea de la adquisición de habilidades de base, antes de intentar habilidades más complejas (Arend, 1980a, 1980b; Magill \& Anderson, 2013), citados por Anderson \& Mayo (2015). 


\section{Objetivo}

Indagar investigaciones relacionadas con la especialización y diversificación temprana y cómo esto puede incidir en el desarrollo de cualidades bio-motoras. Específicamente, se buscó sintetizar algunos principios de ésta revisión, para orientar la tarea del entrenador de jóvenes deportistas en la preparación física. De la misma manera, se busca determinar los riesgos físicos y psicosociales relacionados a la especialización temprana, y sí, esta es garantía del éxito para llegar al deporte de élite.

\section{METODOLOGÍA}

El presente trabajo es una revisión sistemática, la cual para Ruíz \& cols. (2001) "sintetiza el análisis retrospectivo de la información recolectada en diferentes estudios hechos por distintos investigadores". Por tanto, se buscaron artículos que permitieran desarrollar la situación problema, para el presente caso el entrenamiento multilateral de jóvenes acorde a su progreso humano y biológico.

Para la búsqueda sistemática de la literatura de artículos científicos, se utilizaron las bases de datos SPORTDISCUS y Google Scholar, de las que hubo un total de 103 resultados, de los que fueron seleccionados 89 , que fueron publicados entre enero de 2007 y diciembre de 2017 y que son los artículos de texto completo (full text). Adicionalmente, se filtró a publicaciones académicas para un total de 79 y únicamente se tuvieron en cuenta los escritos en inglés (66), de los cuales 2 se repitieron y sólo se consideraron 15 dentro de la investigación que involucraron parámetros psicosociológicos, pedagógicos, motrices y físicos.

Como se muestra en la tabla 1 , se distinguieron varias categorías de análisis, en cuanto al impacto del entrenamiento en los jóvenes. 


\begin{tabular}{|c|c|c|}
\hline \multicolumn{3}{|c|}{$\begin{array}{c}\text { TABLA } 1 \text { PRINCIPALES ARTÍCULOS SOBRE EL ENTRENAMIENTO EN NIÑOS, BENEFICIOS Y } \\
\text { DESVENTAJAS DE LA ESPECIALIZACIÓN TEMPRANA }\end{array}$} \\
\hline CATEGORÍAS & AUTOR Y AÑO & OBSERVACIONES \\
\hline \multirow{5}{*}{$\begin{array}{l}\text { LESIONES } \\
\text { DEPORTIVAS }\end{array}$} & $\begin{array}{l}\text { Osorio, C. \& } \\
\text { cols., } 2007 .\end{array}$ & $\begin{array}{l}\text { Se mencionan las lesiones deportivas a nivel global. } \\
\text { Posteriormente lo discriminan por género y las lesiones } \\
\text { en diversas partes del cuerpo a causa del deporte. } \\
\text { Mencionan de igual manera que los niños son una de } \\
\text { las poblaciones más vulnerables y con tasas más altas } \\
\text { de lesiones. }\end{array}$ \\
\hline & Brenner, J., 2007 & $\begin{array}{l}\text { Habla de las causas del deporte organizado y temprano } \\
\text { en niños. Dentro de estas mencionan el } \\
\text { sobreentrenamiento, las lesiones por sobreuso y el } \\
\text { agotamiento y como estos podrían incidir en la corta } \\
\text { duración en el deporte o en un estilo de vida activo. }\end{array}$ \\
\hline & $\begin{array}{l}\text { Read, P., \& cols. } \\
2016 .\end{array}$ & $\begin{array}{l}\text { En el Reino Unido, las organizaciones de fútbol de élite } \\
\text { han adoptado un enfoque de especialización temprana, } \\
\text { basados en un denominado "Elite Player Performance } \\
\text { Plan" (plan de rendimiento para el deportista de élite". } \\
\text { El principio de este programa es aumentar las } \\
\text { oportunidades de capacitación a través de un marcado } \\
\text { aumentos de las horas de entrenamiento en campo. Sin } \\
\text { embargo, la acumulación de altas horas de } \\
\text { entrenamiento no garantiza el éxito, y adicionalmente y } \\
\text { el impacto de un aumento tan significativo en el } \\
\text { volumen de entrenamiento para los atletas jóvenes que } \\
\text { están experimentando una variedad de procesos de } \\
\text { crecimiento y maduración, podría aumentar el riesgo de } \\
\text { lesiones. }\end{array}$ \\
\hline & $\begin{array}{l}\text { Hall, R., \& cols., } \\
2015 .\end{array}$ & $\begin{array}{l}\text { El estudio compara niñas deportistas, por un lado, las } \\
\text { que practican varios deportes de manera simultánea y } \\
\text { por el otro, las niñas que se especializan en un solo } \\
\text { deporte. Encontrando una mayor incidencia de lesiones } \\
\text { en el primer grupo. }\end{array}$ \\
\hline & $\begin{array}{l}\text { White, J \& } \\
\text { Oatman, D., } \\
2009 .\end{array}$ & $\begin{array}{l}\text { En años anteriores, los niños solían competir o } \\
\text { participar en varios deportes a lo largo de su desarrollo, } \\
\text { esto mediado principalmente por los factores } \\
\text { medioambientales. El curso de este fenómeno ha } \\
\text { cambiado, ya que desde que son muy pequeños, tanto } \\
\text { los padres como los entrenadores deportivos piensan } \\
\text { en la especialización temprana como la vía rápida para } \\
\text { una futura carrera deportiva. Por tanto, el artículo } \\
\text { pretende analizar los beneficios y consecuencias que la } \\
\text { especialización temprana. }\end{array}$ \\
\hline
\end{tabular}




\begin{tabular}{|c|c|c|}
\hline \multirow[t]{4}{*}{$\begin{array}{l}\text { VARIOS DEPORTES } \\
\text { (O ENTRENAMIENTO } \\
\text { MULTILATERAL) VS } \\
\text { ESPECIALIZACION } \\
\text { DEPORTIVA: }\end{array}$} & $\begin{array}{l}\text { Baker, J. \& cols., } \\
2009 .\end{array}$ & $\begin{array}{l}\text { Este documento trata de aclarar el debate entre la } \\
\text { especialización temprana y la diversificación temprana, } \\
\text { basado en la evidencia científica. Alternativamente, } \\
\text { algunos investigadores han propuesto que un enfoque } \\
\text { de diversificación temprana no es una desventaja para } \\
\text { los deportistas en la adquisición de experiencia } \\
\text { deportiva, y es importante para el desarrollo de } \\
\text { intrínseca motivación y la transferencia de habilidad. Sin } \\
\text { embargo, la evidencia presentada no se muestra } \\
\text { contundente hacia alguno de los enfoques. }\end{array}$ \\
\hline & Malina, R., 2010. & $\begin{array}{l}\text { Este artículo de revisión considera las raíces, } \\
\text { consecuencias y la limitación en los datos, sobre la } \\
\text { efectividad de especialización en el deporte. También } \\
\text { rescata el hecho de que un número importante de } \\
\text { entrenadores, padres y niños consideran que la mejor } \\
\text { manera de producir atletas de élite jóvenes, radica en el } \\
\text { hecho de que se dediquen a un solo deporte desde } \\
\text { temprana edad y jugarlo prácticamente todo el tiempo. } \\
\text { Adicionalmente, resaltan múltiples motivos que llevan a } \\
\text { especializar a los niños, dentro de los que se destacan } \\
\text { la búsqueda de becas deportivas. }\end{array}$ \\
\hline & $\begin{array}{l}\text { Ginsburg, R. \& } \\
\text { cols., } 2014 .\end{array}$ & $\begin{array}{l}\text { El estudio consiste en una encuesta realizada a } \\
\text { jugadores de Béisbol profesional, en la cual se les } \\
\text { preguntaba la edad en la que se especializaron en su } \\
\text { deporte. Dentro de los resultados que encontraron } \\
\text { están, que solo el } 25 \% \text { de los encuestados se } \\
\text { especializaron antes de los } 12 \text { años y que la media de } \\
\text { edad de especialización se encontró en los } 15 \text { años. Por } \\
\text { otro lado, el resultado más significativo fue que los que } \\
\text { se especializaron después de esas edades, tuvieron más } \\
\text { opciones de encontrar becas deportivas. Lo que este } \\
\text { estudio refleja es que la diversificación temprana } \\
\text { incrementa las posibilidades de éxito a nivel } \\
\text { universitario y profesional. }\end{array}$ \\
\hline & Street, S., 2010. & $\begin{array}{l}\text { En la actualidad hay más niños participando en el } \\
\text { deporte competitivo y muchos de ellos a edades muy } \\
\text { tempranas como parte de la especialización deportiva. } \\
\text { De ellos han surgido algunas preocupaciones en torno } \\
\text { al desarrollo de estos niños, ya que se ha incrementado } \\
\text { el riesgo a nivel físico y psicosocial. }\end{array}$ \\
\hline
\end{tabular}




\begin{tabular}{|c|c|c|}
\hline $\begin{array}{l}\text { VARIOS DEPORTES } \\
\text { (O ENTRENAMIENTO } \\
\text { MULTILATERAL) VS } \\
\text { ESPECIALIZACION } \\
\text { DEPORTIVA: } \\
\text { (continuación) } \\
\end{array}$ & $\begin{array}{l}\text { Cote y cols., } \\
2009 .\end{array}$ & $\begin{array}{l}\text { La propuesta de los autores para este documento es de } \\
\text { proponer siete principios con respecto al rol de la } \\
\text { diversificación temprana o "sampling" y el juego } \\
\text { deliberado, en oposición a la especialización temprana, } \\
\text { en la promoción de la participación continua y } \\
\text { rendimiento de élite en el deporte. }\end{array}$ \\
\hline & $\begin{array}{l}\text { Goodway y } \\
\text { Robinson, } 2015 .\end{array}$ & $\begin{array}{l}\text { El estudio examina la discusión entre la especialización } \\
\text { temprana y la diversificación temprana, desde una } \\
\text { perspectiva de maduración física y desarrollo motor. } \\
\text { Además, analiza los riesgos de la especialización } \\
\text { temprana como, las lesiones por sobreuso y las } \\
\text { preocupaciones sobre el crecimiento a largo plazo. Y } \\
\text { defiende la diversificación temprana como la base } \\
\text { amplia para la adquisición de habilidades motrices } \\
\text { fundamentales para aplicar en diferentes deportes. }\end{array}$ \\
\hline \multirow{2}{*}{$\begin{array}{c}\text { FACTORES } \\
\text { PSICOLÓGICOS: }\end{array}$} & Weiss, M. 2015. & $\begin{array}{l}\text { En esta revisión examina los beneficios y las } \\
\text { desventajas de especialización temprana desde } \\
\text { perspectivas multidisciplinarias. } \\
\text { Académicos en historia del deporte, aprendizaje motor, } \\
\text { desarrollo } \\
\text { psicología del deporte, desarrollo motor, entre otros, } \\
\text { revisaron teorías, investigaciones empíricas y estudios } \\
\text { de casos que abordan } \\
\text { pros y contras de la especialización temprana frente a } \\
\text { la práctica de múltiples deportes. Dentro de ese } \\
\text { contexto abordan temas como la } \\
\text { adquisición de habilidades, maduración biológica, } \\
\text { autoestima, lesiones, aptitud aeróbica y la capacidad de } \\
\text { mantenerse activos físicamente de por vida. }\end{array}$ \\
\hline & $\begin{array}{l}\text { Nunomura, M \& } \\
\text { cols. (2009). }\end{array}$ & $\begin{array}{l}\text { El estudio se centra en el punto de vista de } \\
\text { entrenadores de gimnasia artística, de deportistas en } \\
\text { desarrollo y la actitud de los gimnastas hacia la } \\
\text { competencia. Y resalta la preocupación de los } \\
\text { entrenadores en cuanto a la motivación, la capacidad de } \\
\text { los atletas para establecer sus propios objetivos y } \\
\text { proporcionar recompensas extrínsecas, tomando esta } \\
\text { última como una herramienta para mantener los } \\
\text { deportistas comprometidos. Sin embargo, hay que } \\
\text { saber dosificar esas recompensas porque si no esto } \\
\text { llevaría a perder el placer de la participación y restaría } \\
\text { valor a la capacidad del deporte de dar forma a valores } \\
\text { positivos. }\end{array}$ \\
\hline
\end{tabular}




\begin{tabular}{|c|c|c|}
\hline & $\begin{array}{l}\text { Wall \& Coté, } \\
2007 .\end{array}$ & $\begin{array}{l}\text { El estudio busca confirmar o negar la hipótesis de que } \\
\text { los niños que los niños que suelen abandonar los } \\
\text { deportes, para el presente caso el hockey sobre hielo, } \\
\text { suelen practicaron menos variedad de deportes en } \\
\text { edades tempranas, tuvieron menor oportunidad de } \\
\text { juego deliberado y gastaron más tiempo en la práctica } \\
\text { deliberada de un deporte especifico. Para poder dar } \\
\text { respuesta a las hipótesis, se efectuó una encuesta a } \\
\text { padres de los niños que aun practica deporte y de } \\
\text { algunos que lo abandonaron. }\end{array}$ \\
\hline PEDAGOGÍA & Hastie, P., 2015. & $\begin{array}{l}\text { El estudio examina la literatura acerca de las } \\
\text { perspectivas pedagógicas en la especialización } \\
\text { temprana y lo dividen en dos fases. La primera una serie } \\
\text { de interrogantes de sentido común examinados desde } \\
\text { una perspectiva pedagógica y segundo, una agenda } \\
\text { investigativa que permita responder dichos } \\
\text { interrogantes. }\end{array}$ \\
\hline
\end{tabular}

\section{REVISIÓN SISTEMÁTICA}

A continuación, se presentarán las visiones de algunos autores sobre los beneficios y desventajas de la especialización deportiva temprana y los efectos positivos del entrenamiento multilateral o diversificación temprana, la revisión se efectúa, por medio de las siguientes categorías de análisis:

- Lesiones deportivas.

- Entrenamiento multilateral vs especialización deportiva.

- Factores psicológicos.

- Pedagogía.

\section{Posibilidad de lesiones deportivas}

En un estudio para determinar la predisposición a la lesión de dolor patelo femoral, se examinaron 546 mujeres deportistas de las cuales, 357 practicaban varios deportes y las otras 189, realizaban, 66 baloncesto, 57 fútbol, y 66 voleibol.

Las deportistas fueron incluidas debido a su diagnóstico de dolor patelofemoral (PFP) en el examen físico. Las pruebas consistieron en una historia estandarizada y un examen físico administrado por el médico para determinar la presencia de PFP. Se encontró que la especialización temprana en deportistas adolescentes, está asociada con el incremento en el riesgo de dolor anterior de la rodilla y otras alteraciones, en comparación con las deportistas que practican varios deportes.

Adicionalmente en el mismo estudio (Fransen J, Pion J, Vandendriessche J, y cols, 2012), citados por Randon y cols. (2015), afirman que la especialización deportiva puede estar relacionada con la reducción y la adquisición de las habilidades y competencias motrices. La diversidad reducida en las actividades de los jóvenes adolescentes podría quebrantar el desarrollo de una 
amplia gama de habilidades motrices comprensivas que se obtienen a través de la participación diversificada en deportes.

La incidencia de lesiones por sobreuso en deportistas jóvenes, ha crecido de forma paralela con la participación de los mismos en los deportes. Hasta el $50 \%$ de todas las lesiones vistas en medicina deportiva en niños, están relacionadas al sobreuso (Dalton, 1992), citado por Randon y cols. (2015). El riesgo de las lesiones por sobreuso es más importante en deportistas niños/adolescentes por múltiples razones, afirman Maffuli (1992) y Carter (1988), citados por Randon y cols. (2015). Dentro de las principales razones de este fenómeno se encuentra, que los huesos en crecimiento de los jóvenes deportistas no pueden soportar tanto stress como los huesos maduros de los adultos.

En el mismo sentido, en un estudio publicado por Brenner (2007), se menciona el peligro potencial de las lesiones por sobreuso en niños y adolescentes, generalmente asociadas a la especialización temprana. El autor define la lesión por sobreuso como un daño micro traumático al hueso, musculo o tendón, que ha sido sometido a un stress repetitivo sin el suficiente tiempo para curar o sin someterse al proceso de reparación natural.

Según el autor, las lesiones por sobreuso, son los factores etiológicos más comunes en niños y adolescentes deportistas, representan hasta el $50 \%$ del total de lesiones. Las lesiones por sobreuso son daños microtraumáticos en el hueso, músculo o tendón, que han sido sometidos a un stress repetitivo, sin el tiempo suficiente para sanar o sin someterse al proceso de reparación natural. La incidencia de lesiones por sobreuso en deportistas jóvenes, tiene un crecimiento paralelo con la participación en deportes.

El autor agrega que los deportistas que practican varios deportes tienen mayor probabilidad de mantener una vida activa por más tiempo, y la capacidad de disfrutar más la actividad física, evitando de la misma manera, las lesiones por: sobreuso, sobreentrenamiento y agotamiento.

Para Osorio (2007), el 80\% de las lesiones sufridas durante la práctica deportiva comprometen los tejidos blandos, tales como músculos, tendones, ligamentos y articulaciones. De igual forma, agrega que entre el 30 a $50 \%$ de las lesiones deportivas son causadas por uso excesivo de tejidos blandos, y que la tasa más alta de lesiones asociadas al deporte y la recreación, ocurre en personas de 10 a 14 años.

Por lo tanto, para Read \& cols. (2016), los deportistas deben ser conscientes de las posibles implicaciones negativas de la especialización deportiva temprana y deberían evitar exposiciones repetidas únicamente a habilidades específicas del deporte, lo que puede aumentar el riesgo de lesiones y limitar la mejora de las habilidades motoras. Además, una comprensión de los cambios fisiológicos asociados con los períodos clave de desarrollo durante el crecimiento de un niño, y cómo esto afecta al movimiento, habilidades y el riesgo de lesión, es necesaria para diseñar efectivamente programas de desarrollo de fútbol a largo plazo. Adicionalmente, el estrés físico asociado con el entrenamiento, también ha demostrado relaciones con lesiones en futbolistas juveniles de élite, y concluyen, que la participación intensa en un solo deporte como el fútbol antes de la maduración física también, puede aumentar el riesgo de lesiones por sobreuso. 


\section{Aspectos físico-motrices relacionados a especialización temprana vs entrenamiento multilateral o diversificación temprana}

El "Poder de la Ley de Práctica" estipula que uno aumenta su nivel de habilidad más significativamente durante la fase inicial de aprendizaje, afirman Baker (2003) y Purcell (2007), citados por Street (2010). En general, para que un individuo obtenga el siguiente nivel de maestría, ese individuo debe pasar más tiempo en la práctica enfocado al objetivo. Las horas deben ser acumuladas durante el período de desarrollo biológico y cognitivo apropiado. Aproximadamente, a los 6-7años de edad, el seguimiento de objetos en movimiento es maduro aproximadamente a los 7-8 años de edad, la postura y el equilibrio son más controlables y en los años preadolescentes la atención selectiva madura con el uso de estrategias complejas de memoria, Stricker (2002), citado por Street (2010).

Para White y Oatman (2009), a pesar de que la especialización temprana, genera algunos beneficios, este fenómeno puede generar unas ganancias a nivel motriz, debido a que competir en varios deportes a temprana edad, puede contribuir al adecuado crecimiento y desarrollo del niño. Mejorará sus habilidades multilaterales, generará mayor autoestima, crecerá física y emocionalmente y evitará el agotamiento por los deportes en la adolescencia. Competir en una amplia variedad de deportes en esas edades puede ayudar a las habilidades multilaterales futuras. El entrenamiento multilateral desarrolla cualidades biomotoras, entendidas como fuerza, velocidad, resistencia, flexibilidad y equilibrio.

Coté y cols. (2009) demostraron, que los deportistas élite participaron en varios deportes durante su infancia. Los deportistas describieron que las actividades durante la niñez se caracterizaban por el juego deliberado, más que en la práctica deliberada. Los autores definen como juego deliberado, las actividades que son divertidas, pero que no contribuyen al desarrollo de la experticia deportiva.

Algunos estudios sugieren que los inconvenientes de la especialización temprana e involucrarse en actividades de entrenamiento intenso a temprana edad, es el hecho que puede limitar el desarrollo de habilidades sociales (Baker, 2009). De igual forma, agrega que las habilidades y la condición física que se ganan a través de la práctica de varios deportes en la niñez, se pueden transferir a la etapa de especialización deportiva.

Según Malina (2010), varios son los motivos que hacen que tanto padres, como niños (especialmente los primeros), opten por la especialización a temprana edad. Dentro de los que se resaltan: conseguir una beca deportiva para estudios, búsqueda de contratos deportivos a nivel profesional y patrocinios con empresas de artículos deportivos. Sin embargo, para el autor, la especialización temprana no está exenta de riesgos. De los cuales menciona: aislamiento social; centrarse en un solo deporte y el compromiso de tiempo asociado puede fomentar el aislamiento de los compañeros de edad y sexo, especialmente, durante la adolescencia, y puede alterar las relaciones con sus compañeros, padres y familia. También, se menciona la sobre dependencia. Las vidas de los jóvenes atletas de élite tienden a estar altamente reguladas. Esto puede fomentar la dependencia excesiva de otros y en muchos casos pérdida de control de lo que está sucediendo en la vida. Agotamiento o "burnout", se asocia con las 
percepciones de atleta, que él o ella no pueden cumplir con las demandas físicas o psicológicas puestas sobre él o ella. Manipulación, porque al sumergir a un joven en un mundo complejo regulado por adultos, puede llegar a ser controlado por algunos adultos con intereses económicos. El crecimiento y la maduración se pueden ver comprometidos, algunas complicaciones en el crecimiento y la maduración ocasionalmente son atribuidas al entrenamiento deportivo intensivo temprano, aunque no hay evidencia contundente al respecto.

Ginsburg y cols. (2014) destacan beneficios relacionados con la práctica deliberada, vinculada con la diversificación temprana, debido a que brinda al niño la oportunidad de experimentar con diferentes movimientos y tácticas mientras aprenden a improvisar y responder estratégicamente en situaciones atléticas. Además, la evidencia sugiere que los jugadores que se especializan más tarde, no solo logran niveles similares de experticia con relación a los deportistas que se especializan temprano, sino que tienen mayor éxito atlético.

En su estudio manifiestan que, aunque la mayoría de deportistas se involucraron con el deporte aproximadamente a los 6 años, no fue sino hasta la adolescencia (alrededor de los 15 años) que se dedicaron exclusivamente a uno. Los datos indican que la mayoría de los atletas mantuvieron la participación en otros deportes hasta edades avanzadas, lo que sugiere que: la diversificación temprana es un método viable y un camino exitoso para llegar al nivel profesional. La muestra mostró, que cuando se compararon los antecedentes deportivos de atletas más altamente calificados con atletas menos capacitados, los atletas altamente calificados típicamente informan que participan en un mayor número o en un volumen mayor (en términos de horas totales de participación) en otros deportes.

Para Goodway \& Robinson (2015), el uso constructivo del deporte juvenil debe ser una parte importante de la educación de cada niño y un medio a través del cual pueden ocurrir procesos clave de desarrollo, desde una perspectiva de crecimiento y desarrollo motor, el desarrollo de la competencia motriz en un amplio el rango de habilidades motrices fundamentales es clave para una trayectoria de desarrollo positiva.

Y, aunque la especialización deportiva, puede tener algunos beneficios, los riesgos asociados con las lesiones y el crecimiento a largo plazo, las altas tasas de agotamiento y el hecho de que los calendarios de práctica temprana e intensa no diferencian a los atletas élite de los no élite, proporciona evidencia para apoyar que la base del desarrollo de las habilidades motrices fundamentales que se aprende a través de la diversificación deportiva, es necesaria e importante antes de la especialización deportiva. Por lo tanto, la diversificación deportiva en los años de juventud formativa es claramente superior a la especialización deportiva temprana.

\section{Consideraciones a nivel psicológico}

Adicionalmente, a los efectos físicos de los programas de especialización temprana, también, se encuentran consecuencias psicológicas en los jóvenes deportistas, como lo es el síndrome de agotamiento o sobre entrenamiento. El síndrome de sobre entrenamiento puede definirse como: una "serie de cambios psicológicos, fisiológicos y hormonales que resultan en un menor desempeño deportivo". Las manifestaciones 
más comunes pueden incluir dolor muscular - articular crónico, cambios en la personalidad, ritmo cardíaco en reposo elevado y disminución del rendimiento deportivo. El niño deportista también, puede tener fatiga, falta de entusiasmo por la práctica o la competencia, o dificultad para completar satisfactoriamente las rutinas habituales. El agotamiento debe ser reconocido como una secuela grave del síndrome de sobre entrenamiento, reconocen Small (2002) y Budgett (1998), citados por Randon y cols. (2015).

De igual forma, hay que resaltar que muchos deportistas deciden abandonar su actividad como efecto colateral de la especialización temprana. Dentro de las razones más frecuentes para hacerlo, se encuentran: el conflicto de intereses con otras actividades, demasiado tiempo de compromiso, pérdida de tiempo de juego, demasiada presión, asuntos inherentes a la competencia y pérdida de la diversión, siendo esta última la razón más frecuente (Wall \& Coté, 2007).

En este mismo estudio, que cual fue efectuado con jugadores de hockey sobre hielo, concluyen los autores, que un aspecto que diferencia de manera significativa a los deportistas que abandonan, de los que no, es el hecho de que los primeros, iniciaron el entrenamiento por fuera del hielo a más temprana edad (12 y 13 años), lo que sugiere una forma de especialización temprana, lo que soporta el postulado que la participación temprana en programas específicos de entrenamiento, puede en última instancia socavar la motivación para continuar en el deporte.

Para Weiss (2015), al aplicar conceptos y extrapolar los hallazgos de los estudios sobre el aprendizaje motor, la psicología social del deporte, el crecimiento, el desarrollo motor y la fisiología del ejercicio en niños, se contribuye a los méritos de desarrollar una amplia base de habilidades motrices fundamentales durante la infancia. Cuando las habilidades se enseñan dentro de un entorno de apoyo y un ambiente orientado al dominio, es probable que los niños logren autopercepciones positivas, una orientación motivacional intrínseca, competencias a nivel deportivo y niveles de condición física, que les permitan ser físicamente activos y acumular beneficios holísticos de salud en la adolescencia y la edad adulta.

Hay deportes en donde la especialización temprana y las altas cargas de entrenamiento son la norma. Como es el caso de la gimnasia artística, en donde, por ejemplo, en Brasil, los niños pueden competir oficialmente desde los siete años. Por tanto, ha surgido una crítica dirigida al deporte juvenil y es su potencial como fuente de estrés, debido a que la competencia aumenta las expectativas tanto del atleta como del círculo de atletas. En la competencia juvenil, el conjunto de presiones externas que el atleta se autoimpone, puede producir un "punto de inflexión" que conduce a un atleta desmotivado a que sufra agotamiento y abandone por completo. Si bien el estrés, es una parte normal de la vida, independientemente de la edad, con los jóvenes, sin embargo, existe un deber de cuidado para garantizar que las tensiones encontradas sean manejables sintetizan Nunomura \& cols. (2009), apoyados en: De Rose Jr., (2002); Weinberg \& Gould (2001); Bompa (2000), Hedstrom, Gould (2004); De Rose Jr., Butcher \& Linder (2004). 


\section{Elementos pedagógicos}

Para Hastie (2015), desde la perspectiva pedagógica hay una serie de elementos que deberían considerarse por parte de los entrenadores (y los centros a cargo de los mismos) que dirigen los programas de entrenamiento de los deportistas que se especializan temprano. Por tal motivo orienta su investigación con una serie de supuestos como: Ios niños que se especializan temprano deberían recibir entrenamiento por entrenadores con mayores conocimientos, esos entrenadores deberían tener planes de entrenamiento más sofisticados, y que, conforme a la práctica deliberada, los deportistas que se especializan temprano puedan experimentar climas motivacionales que sean más orientados a consecución de tareas. Sin embargo, no pudo dar respuestas definitivas a los mismos, ya que muy pocos estudios reportaron estados de especialización en sus participantes.

Otra orientación que se le dio al estudio permitió hallar suficiente evidencia que sugiere que la participación en un solo deporte no es el camino para llegar al deporte de elite, de hecho, los resultados mostraron que los deportistas elite se especializaron tarde, entrenaron menos en la niñez y diversificaron con más deportes.

\section{META ANÁLISIS}

Se aprecia que la especialización temprana a diferencia de la diversificación temprana o entrenamiento multilateral, tiene una gran incidencia en la aparición de lesiones por sobreuso. Uno de los factores relacionados es el poco desarrollo de los tejidos (huesos, músculos y tendones), los cuales no pueden soportar el stress dado por el entrenamiento. Otro de los factores comunes es la exposición repetida de movimientos 0 habilidades específicas de cada deporte.

También se resalta el hecho de que los niños que participan o practican varios deportes incrementan la probabilidad de mantener un estilo de vida activo en la adultez, tienen mayor capacidad de disfrute hacia la actividad física y disminuyen el riesgo de las mencionadas lesiones por sobreuso o sobreentrenamiento y mitigan el agotamiento o abandono del deporte.

Desde el aspecto físico-motriz, el entrenamiento multilateral proporciona las bases o cimientos de las habilidades, por tanto que en las edades inferiores a 12 años, al encontrarse las fases iniciales y de desarrollo del aprendizaje motriz, se les debería proporcionar diversificación en actividades físicas o la posibilidad de practicar varios deportes, ya que mejorarían sus habilidades multilaterales y se verían potenciados muchos más elementos, tanto a nivel físico, emocional, social y psicológico, además de contribuir a un desarrollo y crecimiento apropiado. No obstante, aunque la especialización temprana genera algunos beneficios, el competir en varios deportes ayuda a las habilidades multilaterales futuras, la transferencia de habilidades entre deportes y desarrollar cualidades biomotoras y/o capacidades físicas.

A pesar de que, uno de los motivos principales para iniciar a un niño en la especialización deportiva temprana, es la búsqueda de la élite deportiva o el deporte profesional, no hay garantía de que este fenómeno pueda garantizar el éxito futro. De modo contrario, se ha encontrado en varios estudios que varios deportistas élite manifestaron que participaban en varios deportes en su infancia y que muchas de las 
actividades de las que eran participe, se identificaban más con el juego deliberado. Al mismo tiempo, los estudios muestran que los deportistas que suelen especializarse más adelante (alrededor de los 15 años), amplían las posibilidades del éxito deportivo.

Los hallazgos a nivel psicológico, del impacto negativo de la posible especialización temprana, son contundentes. Es muy frecuente, encontrar niños con síndrome de agotamiento o sobreentrenamiento, lo que repercute de manera directa y negativa en el rendimiento deportivo. Esto ligado a la falta de motivación hacia los entrenamientos o competencias deportivas y pérdida del disfrute. Lo que a mediano o largo plazo podría desencadenar en abandono de la actividad.

El campo pedagógico respalda los hallazgos encontrados en las otras categorías, porque se halló que, la participación y competencia en un solo deporte no es el camino para llegar a la élite deportiva. Por el contrario, los resultados mostraron que los deportistas élite, manifiestan haberse especializado tarde, entrenaron menos en etapas tempranas y tuvieron mayor diversificación deportiva. Del mismo modo, nos hace plantearnos varios interrogantes acerca del nivel de capacitación, en materia de entrenamiento deportivo, fisiología y psicología del desarrollo, entre otros, que requiere un profesional que esté a cargo de los niños que se están especializando temprano, y si, este a su vez, está creando un ambiente motivacional adecuado para ellos.

\section{CONCLUSIONES}

Los datos de esta revisión sugieren que una propuesta de entrenamiento deportivo, caracterizada por el entrenamiento multilateral, basado en un enfoque de diversión y gran variedad de actividades deportivas durante la niñez, podrían mermar los efectos físicos y psicosociales asociados con la especialización temprana. También, se considera que la diversificación temprana conlleva a la experticia deportiva, debido a la motivación intrínseca que surge del disfrute, la diversión y la competencia que se experimenta con la participación deportiva. Asimismo, sugiere que las habilidades y el acondicionamiento físico desarrollados a través de la participación diversificada puede transferirse a la especialización deportiva más adelante.

La especialización temprana no es el único camino, ni una norma, para llegar a la élite deportiva o al deporte profesional, por el contrario, se encontró que muchos de los deportistas abandonan en el camino. De igual forma, el éxito en la niñez, no garantiza el éxito más adelante en la élite deportiva. Sin embargo, hay deportes donde la especialización temprana es requerida, debido a que la cumbre del rendimiento se encuentra antes de la maduración completa, como, por ejemplo, la gimnasia artística. 


\section{REFERENCIAS BIBLIOGRÁFICAS}

Anderson, D., \& Mayo, A. (2015). A Skill Acquisition Perspective on Early Specialization. Sport Kinesiology Review. 4 (3), 239.

Baker, J., Cobley, S., \& Fraser, J. (2009). What do we know about early sport specialization? Not much!, Rev Routledge. 20 (1), 80.

Brenner, J. (2007). Overuse Injuries, Overtraining, and Burnout in Child and Adolescent

Athletes. Rev Pediatrics. 119 (6), 1243.

Coté, J., Horton, S., MacDonald, D., \& Wilkes, S. (2009). The Benefits of Sampling Sports During Childhood. Physical \& Health Education Journal. 74 (4), 7.

Ginsburg, R., Smith, S., Danforth, N., Ceranoglu, A., Durant, S., Kamin, R., Robin, L., \& Masek, B. (2014). Patterns of Specialization in Professional Baseball Players. Journal of Clinical Sport Psychology. 8(3), 263,271.

Goodway, J., \& Robinson, L. (2015). Developmental Trajectories in Early Sport Specialization: A Case for Early Sampling from a Physical Growth and Motor Development Perspective. Human Kinetics (kinesiology Review). 4 (3), 275.

Hall, R., Barber, K., Hewett, T., \& Myer, G. (2015). Sport Specialization's Association With an Increased Risk of Developing Anterior Knee Pain in Adolescent Female Athletes. Journal of Sport Rehabilitation. 24(1), 32-33.

Hastie, P. (2015). Early Sport Specialization from a Pedagogical Perspective. . Kinesiology Review. Human Kinetics, 4 (3), 300.

Jones, L., Petlichkoff, L. (2008). Early Sport Specialization Versus Quality Physical Education. Chronicle of Kinesiology \& Physical Education in Higher Education. 19 (2), 5.

Malina, R. (2010). Early Sport Specialization: Roots, Effectiveness, Risks. Current Sports Medicine Reports (American College of Sports Medicine). 9(6). 365-369.

Nunomura, M., Okade, Y., \& Tsukamoto, M. (2009). Competition and Artistic Gymnastics: How to Make the Most of This Experience. International Journal of Sport and Health Science. 7, 43.

Osorio, J., Clavijo, M., Arango, E., Patiño, S., y Gallego I.(2007). Lesiones deportivas, Rev latreia, 20(2), 168.

Read, R., Oliver, J., De Ste Croix, M., Myer, G., y Lloyd, R. (2016). The scientific foundations and associated injury risks of early soccer specialization. Journal of Sports Sciences. 34 (24), 2296, 2298,2300.

Ruíz, A., Gómez, C. y Londoño, D. (2001). Investigación clínica: epidemiologia clínica aplicada. Bogotá, Colombia: Centro editorial Javeriano.

Sluder, B., Fuller, T., Griffin, S., Mccray, Z. (2017). Early vs. Late Specialization in Sport. GAHPERD Journal. 49 (3), 10.

Street, S. (2010). The Early Specialization of Youth in Sports. Athletic Training \& Sports Health Care. 2 (6), 256.

Wall, M., \& Coté, J. (2007). Developmental activities that lead to dropout and investment in sport. Rev Routledge. 12(1), 79.

Weiss, M., (2015). "Bo Knows" Revisited: Debating the Early Sport Specialization Question. Kinesiology Review. Human Kinetics 4 (3), 219. 
White, J., \& Oatman, D. (2009). Does Specializing in Team Sports During Childhood Translate Into a College Athletic Career?. Journal of Health, Physical Education, Recreation and Dance. $19,21$. 